\title{
Linx
}

Revue des linguistes de l'université Paris X Nanterre

$12 \mid 2002$

«Comme la lettre dit la vie »

\section{Les îlots textuels dans Sodome et Gomorrhe de Proust}

Carole Tisset

\section{OpenEdition}

Journals

Édition électronique

URL : http://journals.openedition.org/linx/1317

DOI : $10.4000 / \operatorname{linx} .1317$

ISSN : 2118-9692

Éditeur

Presses universitaires de Paris Nanterre

Édition imprimée

Date de publication : 1 octobre 2002

Pagination : 261-268

ISSN : 0246-8743

\section{Référence électronique}

Carole Tisset, "Les îlots textuels dans Sodome et Gomorrhe de Proust », Linx [En ligne], 12 | 2002, mis en ligne le 10 octobre 2012, consulté le 30 avril 2019. URL : http://journals.openedition.org/linx/1317 ; DOI : 10.4000/linx.1317

Ce document a été généré automatiquement le 30 avril 2019

Département de Sciences du langage, Université Paris Ouest 


\title{
Les îlots textuels dans Sodome et Gomorrhe de Proust
}

\author{
Carole Tisset
}

1 Je voudrais ici analyser les différentes formes d'îlots textuels du point de vue de leur forme d'insertion dans le discours du narrateur: éléments typographiques ou non, changements syntaxique et énonciatif ou non ... ainsi que leur rôle dans la fiction : ajouts descriptifs sur la gestuelle ou sur l'intonation ; langage socialement marqué ou non.

2 Nous commencerons par des définitions négatives pour cerner la notion.

3 - L'IT n'est pas un discours rapporté indirectement (DI) sous la forme :

le narrateur écrit que « $\mathrm{X}$ a dit que + subordonnée »

mais il s'en rapproche dans la mesure où le discours du narrateur utilise un élément discursif d'autrui.

4 - L'IT n'est pas du discours rapporté directement (DD) selon la formule :

le narrateur s'efface et rapporte que $\mathrm{X}$ a dit : «P », mais il est voisin puisqu'il fait mention d'un élément discursif autre.

5 - L'IT ne connaît pas les changements de temps et de personne du discours indirect libre (DIL) mais, comme lui, il imbrique deux plans énonciatifs. Nous trouvons dans Sodome et Gomorrhe des discours indirects libres toujours plus difficiles à trouver quand la narration se fait à la première personne :

\begin{tabular}{|l|l|l|}
\hline & (1) & Je venais de me rappeler que depuis de longues semaines j'avais \\
\hline \hline & & oublié d'écrire à ma grand-mère. Que devait-elle penser de moi ? \\
\hline & & « Mon Dieu me disais-je... » (157) \\
\hline
\end{tabular}

6 Nous partirons d'une première définition de l'IT comme fragment de discours second inséré dans le discours premier du narrateur qui en fait la mention et l'usage dans son propre discours (a) ou dans un discours indirect (b). La citation se fait sans reformulation 
comme pour le DD mais n'entraîne ni rupture syntaxique ni rupture énonciative. L'IT est intégré à la pâte narrative, à la syntaxe du discours premier sans être pour autant un DI ou un DIL puisque l'énonciateur signale ce qui ne lui appartient pas. Il s'agit dans les deux cas d'une forme de modalisations autonymiques ${ }^{1}$ puisqu'il est fait mention et usage des mots des autres.

7 a- dans le discours premier : Pour mon poste de secrétaire, c'est « foutu », comme dirait Pierre.

[ E..., «IT », comme dit L]

b- dans un DI: Pierre a dit à propos de son travail que c'était « foutu ».

$\mathrm{E}$ [L (dire) que ... P incluant « IT »]

Chez Proust, l'I.T se présente sous ces deux formes mais également sous d'autres.

\section{I- Un fragment discursif autre dans le discours premier}

1.1 L'IT se caractérise par l'insertion d'un fragment de discours d'autrui, IT, dans le discours du narrateur, dont la source ( $\mathrm{L}$ : locuteur = émetteur second) est dénommée et l'insertion signalée par des guillemets :

(2) Au reste l'ambassadrice de Turquie, comme disait la duchesse de Guermantes (L) qui passa avec moi l'inspection des salons, «faisait bien »(insertion d'un fragment de L = IT) $(60)$

Le narrateur utilise et cite dans son discours la locution « faire bien » tout en en donnant la provenance puisqu'elle n'est pas sienne.

Nous trouvons de nombreux exemples de ce type de citations. En voici quelques-uns :

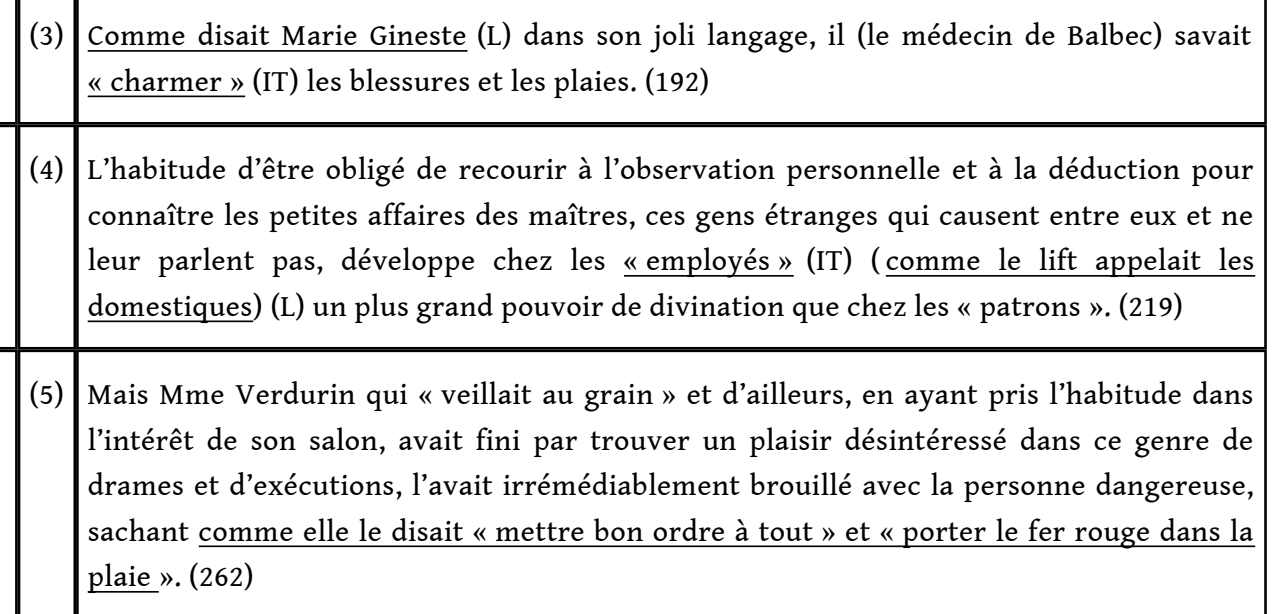

En (5), pour les deux IT de la fin de phrase le narrateur signale explicitement qu'il emploie des expressions de Mme Verdurin. On peut penser que la première citation est si proche du nom de l'émettrice que le narrateur a cru inutile de le signaler. De même en (4) l'opposition claire employés/patrons rend inutile la répétition de la source. L'émetteur dont on emprunte les mots est signalé par la forme « comme dit X ». 
14 1.2 L'IT peut se trouver inséré dans un discours indirect. Le narrateur effectue donc deux opérations. Il intègre les propos du personnage dans son propre discours et donc dans son énonciation (avec les changements de repérages nécessaires) mais aussi avec des reformulations, des interprétations, des synonymies, etc. Le DI est une transformation de l'énonciation du locuteur second. Mais le narrateur tient à signaler que certains termes ne sont pas de lui.

(6) Mais après avoir célébré le «palais " de sa cousine, elle s'empressa d'ajouter qu'elle préférait mille fois «son humble trou». «Ici, c'est admirable pour visiter. Mais je mourrai de chagrin s'il me fallait rester à coucher dans des chambres... (68)

L'exemple (6) contient un IT du type -a- avec le mot "palais » que le narrateur exhibe comme employé par la duchesse de Guermantes, puis un DI sous la forme d'une subordonnée conjonctive introduite par le verbe locutoire "ajouter». L'expression « humble trou » encadrée par des guillemets et utilisée dans le DI signale que l'IT de type -b-, est un fragment du discours de la duchesse, employé par elle et restitué par le narrateur.

(7) C'est ainsi que Mme de Marsantes, quand quelqu'un d'un monde différent entrait dans son milieu, vantait devant lui les gens discrets « qu'on trouve quand on va les chercher et qui se font oublier le reste du temps » comme on prévient sous une forme indirecte un domestique qui sent mauvais que l'usage des bains est parfait pour la santé. (63)

Le DI ne contient qu'un GN introduit par le verbe locutoire évaluatif « vanter ». Ce GN est suivi d'une longue expansion qui forme l'IT $b$ signalant la reprise à l'identique des propos de Mme de Marsantes. L'emploi du présent n'est pas ici une erreur ou un télescopage de deux énonciations puisqu'il a une valeur omnitemporelle.

On remarqua également en (6) que l'IT permet de passer du DI au DD. Il y a continuité du discours second amorcé dans celui du narrateur avec un émetteur identique pour l'IT comme pour le DD. Comme tout DD, il présente les nécessaires changements de repères. On trouve le déictique « ici » et l'embrayeur de 1 ère personne. Les deux types de discours rapportés se trouvent dans deux phrases distinctes. Les guillemets qui encadrent la deuxième phrase indiquent bien qu'il s'agit d'une mention " pure » du discours second. Les guillemets ne jouent pas le même rôle dans la première phrase puisqu'ils permettent d'encadrer le fragment dit par le locuteur second, mentionné comme tel et utilisé par le narrateur. Dans le cas du discours direct, l'interprétation des guillemets est univoque, dans le second cas, elle est équivoque. Comme le narrateur fait usage et mention d'un fragment discursif ne lui appartenant pas, on peut s'interroger sur le degré d'adhésion du narrateur dans l'emploi de ce fragment.

Dans un cas comme dans l'autre, les mots d'autrui semblent rebelles à toute appropriation parce que le narrateur ne veut pas les faire siens tout aussi bien parce qu'ils les acceptent mais souhaite en nommer la source. A l'intérieur d'une narration, dans l'appropriation du discours d'autrui se noue un rapport conflictuel entre ce qui est accepté et acceptable et ce qu'il faut respecter, laisser intact, ne pas dénaturer. Ces «échappés » d'énonciation seconde ont un fort rendement dans la fiction. En effet, le 
discours narrativisé et l'indirect ruinent la mimesis, mais l'IT la restaure. Du coup, les êtres de fiction se voient pourvus d'un parler qui semble résister à l'opération de fiction et entraîner le lecteur vers plus de crédulité.

19 Dans la relation entre l'IT et les autres formes de discours rapporté, nous trouvons dans Sodome et Gomorrhe, deux autres configurations.

\section{III- Autres formes d'IT}

L'îlot textuel n'apparaît pas uniquement dans le discours premier ou dans un discours indirect.

\section{1-L'IT peut être également intégré à un DIL}

(8) Le directeur vint me demander si je ne voulais pas descendre. A tout hasard il avait veillé déjà mon "placement " dans la salle à manger. Comme il ne m'avait pas vu, il avait craint que je ne fusse repris de mes étouffements d'autrefois. Il espérait que ce ne serait qu'un tout petit «maux de gorge » et m'assura avoir entendu dire qu'on les calmait à l'aide de ce qu'il appelait : « le calyptus ». (160)

Le narrateur rapporte dans la première phrase le premier échange avec le directeur de l'hôtel sous la forme d'un DI. Il passe ensuite au DIL. Le pronom anaphorique de troisième personne reprend le GN « directeur ». Les temps employés sont l'imparfait ou le plus-queparfait d'antériorité pour la translation aux temps de la narration. La deuxième personne de politesse, très certainement employée par le directeur est transformée en première personne de la narration autobiographique. Il y a donc superposition des deux voix, transformation et intégration du discours second dans le discours premier. Certains mots employés par le directeur apparaissent entre guillemets et signalent alors la reprise à l'identique. Le premier IT appartient au langage du directeur, parce que c'est une lexie professionnelle, les deux autres parce que ce sont des erreurs. Le directeur ignore manifestement le singulier du terme "maux». Le troisième IT cite une erreur phonologique qui entraîne une mauvaise coupure et la création d'une lexie qui n'existe pas. L'erreur est telle que le narrateur la signale doublement par l'encadrement des guillemets et par l'annonce de l'IT grâce au verbe appeler suivi des deux points, signe précurseur de la citation. Nous avons ici un IT puisque la phrase serait incomplète sans lui mais aussi un effet de citation plus proche du DD parce qu'il y a une rupture énonciative introduite par les deux points.

L'IT intégré au DIL amplifie la voix seconde, lui restitue par bribes ses tics et ses imperfections. Il y a à la fois superposition des voix, polyphonie et mise à distance. De temps en temps, l'énonciateur tient à se démarquer du locuteur. L'IT participe alors d'une distance ironique très perceptible lorsqu'il s'agit de dénoncer les travers langagiers des uns ou des autres. Comme nous sommes dans une fiction, cette dénonciation, cette distance confèrent plus d'authenticité aux personnages puisque leurs défauts langagiers sont épinglés. Le narrateur restitue leur parole jusque dans ses défauts. 


\section{2 - L'IT peut appartenir à un émetteur anonyme} « « tournée ». (248)

Nissim Bernard est myope et confond deux frères jumeaux. Il s'adresse par erreur à celui qui n'aime que les femmes:

(9) «Veux-tu me donner rendez-vous pour ce soir?» Il recevait aussitôt une solide

Le DD cite la phrase employée par Nissim Bernard. Il est suivi d'une phrase contenant un IT de type a- intégré au discours du narrateur. Cet IT vient d'un émetteur anonyme, de la vox populi, et est marqué sociologiquement.

5 Les exemples (8) et (9) nous amènent à faire deux autres distinctions. Non seulement il convient d'observer à quel niveau discursif l'IT est utilisé mais aussi quel type d'émetteur est proposé. En effet, on peut trouver des IT avec émetteur précis ou émetteur anonyme. Nous avions déjà proposé (Tisset, 2000) de faire cette distinction en réservant le terme de « connotation autonymique » pour tout émetteur générique et « îlot textuel » pour tout émetteur spécifique.

Cette dernière distinction, qui porte sur la nature de l'émetteur, est très fréquente chez Proust.

\begin{tabular}{|l|l|l|l|}
\hline (10) & $\begin{array}{l}\text { je prends ...la chose incroyable appelée « correspondance », ...je change ainsi jusqu'à } \\
\text { trois, quatre fois de « voiture »...dans un de ces affreux wagons où on a comme vue, } \\
\text { entre des triangles d'ouvrages dits de « filet », la photographie des principaux chefs- } \\
\text { d'œuvre d'architecture du réseau...j'avais en face de moi, une « vue » de la cathédrale } \\
\text { d'Orléans.. (12-13) }\end{array}$ \\
\hline (11) & $\begin{array}{l}\text { j'allai passer quelques jours à l'«hôtel » comme on dit. Un des garçons d'étage m'était } \\
\text { connu, je lui désignai un curieux petit « chasseur »... (14) }\end{array}$ \\
\hline (12) & c'était l'huissier (qu'on appelait dans ce temps-là « l'aboyeur ») (35) \\
\hline (13) & $\begin{array}{l}\text { M'écartant de l'éblouissante maison de « plaisir », insolemment dressée là malgré les } \\
\text { protestations des familles ... (180) }\end{array}$ \\
\hline
\end{tabular}

\section{Parfois l'IT n'est pas signalé par des guillemets.}

(14) Je fus ennuyé de voir qu'ils étaient presque tous dans la tenue qu'on appelle à Paris smoking. (263)

L'absence de guillemets, peut-être due à une erreur d'édition, est étrange dans la mesure où le terme n'est pas français, qu'il est introduit par le verbe « appeler » qui annonce la citation comme en (8).

On retrouve l'absence de signalement d'un IT dans l'usage d'une métaphore populaire. 

mari qui portait les jupes et la femme les culottes. (46)

Les exemples (9) à (15) utilisent des fragments discursifs dont l'émetteur est tout le monde et s'opposent aux IT suivants dont l'émetteur est dûment signalé :

\begin{tabular}{|l|l|l|l|}
\hline (16) & Il enfila le couloir en feignant de chercher ce que Cottard appelait les « waters ». (268) \\
\hline (17) & $\begin{array}{l}\text { (à propos de Pelléas et Mélisande): « Mais au contraire, c'est un petit chef-d'œuvre » et de } \\
\text { «discuter ». C'était peut-être une habitude de Combray, prise auprès des sœurs de ma } \\
\text { grand-mère (e) qui appelaient cela : « combattre pour la bonne cause »... (207) }\end{array}$ \\
\hline
\end{tabular}

y compris quand le narrateur ne veut pas nommer précisément l'émetteur :

(18) Le prince de Guermantes, au lieu de recevoir sur place le bonsoir du mari d'odette, l'avait aussitôt, avec la puissance d'une pompe aspirante, entrainé avec lui au fond du jardin, mais me dirent certaines personnes, « afin de le mettre à la porte ». (56)

Nous avons donc deux oppositions en fonction de l'émetteur nommé ou générique ( 2 vs 9); trois oppositions en fonction du niveau d'intégration de l'IT : dans le discours du narrateur (3), dans le discours indirect $(6 ; 7)$, dans le discours indirect libre (8); des nuances entre la mention et l'usage selon que l'effet citation est plus ou moins marqué par la présence ou non d'un verbe introducteur, la présence ou non des guillemets, la présence ou non des deux points.

Toutes les combinaisons sont possibles. En (15) l'IT est intégré mais non signalé typographiquement parce qu'il est à la limite de l'allusion intertextuelle. Il apparaît dans un discours indirect dont l'émetteur est signalé : « au ministère ». L'effet produit par l'IT générique est de souligner le décalage entre l'expression mentionnée et son usage. L'IT permet non seulement de mentionner les mots des autres dans son discours, donc de se les approprier mais également de s'en distancer, de les évaluer.

\section{IV- IT et évaluation du dire}

\section{1- Dédoublement du narrateur}

33 Il existe une dernière opposition de contenu dans l'usage des guillemets. Les guillemets permettent au narrateur de s'autoriser l'usage d'une expression qu'il juge malséante ou inadéquate. Il ne s'agit pas d'un émetteur autre puisque le narrateur fait sienne l'expression. Le fragment n'a d'autre source que lui-même mais il s'écarte de la langue habituelle du narrateur. Le fragment entre guillemets fait apparaître un dédoublement du narrateur. Le premier fait un usage normé de la langue mais s'autorise à des écarts qu'il mentionne comme s'ils étaient de quelqu'un d'autre moins soucieux de l'homogénéité du niveau de langue. 


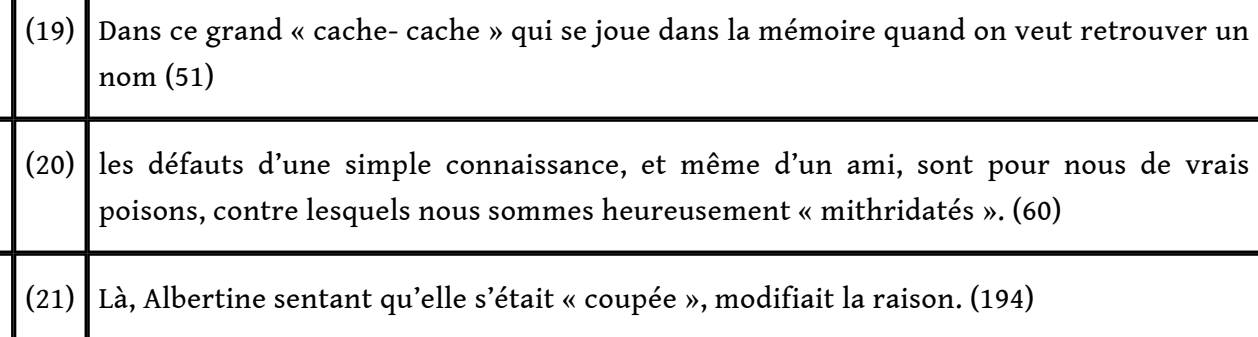

(22)

$\mathrm{Si}$, en effet, la princesse, même s'ils étaient de ses amis, ne les conviaient pas, cela tenait souvent à sa crainte de mécontenter " Palamède » qui les avait excommuniés (48)

23) A cet égard elle fut vite rassurée, ayant appris que le prince n'avait pas laissé entrer Swann et avait eu avec lui « une espèce d'altercation ». Elle ne risquait pas d'avoir à faire publiquement la conversation avec « pauvre Charles » qu'elle préférait chérir dans le privé. (73) familiarité que s'octroie le narrateur et qu'il juge un peu leste ou si c'est une dénomination utilisée par la princesse.

De même, dans l'exemple (5), on ne sait si l'expression "veiller au grain » doit être attribuée à Mme Verdurin donc en IT ou au narrateur donc en « écart modalisé ». 
cet exemple, l'expression " pauvre Charles » est un fragment du discours de Mme de Guermantes car le narrateur le restitue sans le déterminant comme il est d'usage dans une apostrophe. De plus, seule la duchesse peut se permettre d'appeler Swann par son prénom. On s'amuse ici du paradoxe entre l'apitoiement qui transparaît dans l'IT et le soulagement de la duchesse à ne pas parler à Swann. L'opposition vie publique/ vie privée expliquée par le narrateur est en quelque sorte « mimée » par le texte qui oppose mots du narrateur et mots de la duchesse.

Pour le fragment « une espèce d'altercation », ce peut être tout aussi bien une citation en IT, ou une expression jugée inadéquate par le narrateur parce qu'antinomique ou excessive selon qu'on y voit de l'ironie ou non.

(24) C'étaient ce que le langage des hôtels appelait deux courrières et celui de Françoise, laquelle s'imaginait qu'un courrier ou une courrière sont là pour faire des courses, des " coursières".

41 Les guillemets indiquent à la fois une citation de Françoise en IT et un écart avec le mot correct. La citation est utilisée dans une réflexion sur l'étymologie des erreurs lexicales de Françoise. Nous touchons là une autre limite de l'IT.

L'IT se différencie de l' "écart» par la source. L'IT appartient à autrui. Mais il est également une évaluation.

\section{V- L'IT et la citation}

Chez Proust, l'IT entraîne parfois une réflexion métalinguistique sur la langue des autres. Les citations sont alors expliquées ou évaluées. Mais une citation autonyme n'est pas un IT. La condition sine qua non de l'IT est l'usage du fragment cité, son insertion dans le discours du narrateur comme nous l'avons montré en 1 et 2 .

(25) Je crois que son nom était Mme Poussin. Mais nous ne l'appelions jamais entre nous que «Tu m'en diras des nouvelles» (1), car c'est par cette phrase perpétuellement répétée qu'elle avertissait ses filles des maux qu'elles se préparaient, par exemple en disant à l'une qui se frottait les yeux : «Quand tu auras une bonne ophtalmie, tu m'en diras des nouvelles (2); » Elle adressa de loin à maman de longs saluts éplorés, non en signe de condoléance, mais par genre d'éducation. Nous n'eussions pas perdu ma grand-mère et n'eussions eu que des raisons d'être heureux qu'elle eût fait de même. Vivant assez retirée à Combray dans un immense jardin, elle ne trouvait jamais rien assez doux et faisait subir des adoucissements aux mots et aux noms mêmes de la langue française. Elle trouvait trop dur d'appeler « cuiller » la pièce d'argenterie qui versait ses sirops et disait en conséquence « cueiller »; (168)

Avec beaucoup d'humour, le narrateur rapporte le surnom de Mme Poussin qui est une phrase constamment utilisée par cette dame et citée en $\mathrm{DD}(2)$. Ce tic langagier est employé par le narrateur comme un énoncé autonyme employé en usage et en mention puisqu'il est devenu un désignateur rigide (1). 

Mme Poussin, la première occurrence de «cuiller » est orientée sur la prononciation en usage habituellement même si Proust désigne sa référence: "pièce d'argenterie qui versait ses sirops ", comme le ferait un article de dictionnaire. On peut l'analyser comme un IT d'un locuteur générique intégré au discours du narrateur. La difficulté tient au fait que nous sommes dans un discours métalinguistique qui considère le signe linguistique dans sa matérialité comme dans sa signification. Dans la deuxième occurrence entre guillemets il s'agit d'expliquer la prononciation d'un locuteur précis, le mot est employé pour sa matérialité sonore et non pour sa signification. Il s'agit donc d'une citation autonyme. On voit que dans un discours métalinguistique la frontière est bien ténue entre IT et citation, que l'IT ne se différencie que par le rappel de la signification.

Nous avons le même effet de citations fragmentaires à propos de l'idiolecte du liftier et des réflexions qu'il inspire au narrateur :

(25) Mais son langage était défectueux. Je croyais à son ambition parce qu'il disait en parlant du concierge, duquel il dépendait: "Mon concierge ", sur le même ton qu'un homme possédant à Paris ce que le chasseur eût appelé " un hôtel particulier ", eût parlé de son portier. Quant au langage du liftier, il est curieux que quelqu'un qui entendait cinquante fois par jour un client appeler: "Ascenseur ", ne dît jamais luimême qu'" accenseur ». (187)

Le premier terme cité apparait en DD : rupture énonciative avec les deux points et utilisation de l'embrayeur de première personne sous la forme du déterminant possessif. Le locuteur second est nommé et la citation annoncée par le verbe neutre: dire. La deuxième mention est un IT hypothétique intégré au discours du narrateur avec un émetteur désigné comme en (16). Les troisième et quatrième mentions se distinguent par la source mais aussi par l'insertion. L'une est attribuée à un client anonyme représentatif de la norme, l'autre est l'usage du liftier. La première apparaît comme en DD avec une rupture énonciative introduite par les deux points. La seconde appartient à un DI sous la forme classique d'une subordonnée conjonctive complément du verbe dire. Selon les caractéristiques définies plus haut, seule cette dernière occurrence est un IT.

Par effet symétrique, certaines connotations autonymiques ne sont pas signalées comme telles :

(26) Bien vite, le lift, ayant retiré ce que j'eusse appelé sa livrée et ce qu'il nommait sa tunique, apparaissait en chapeau de paille, avec une canne, soignant sa démarche et le corps redressé, car sa mère lui avait recommandé de ne jamais prendre le genre « ouvrier » ou « chasseur ». (63)

Les mots «livrée » et "tunique » ne sont pas encadrés par des guillemets comme " ascenseur » en (25); ils sont entièrement intégrés à la narration et pourtant ils sont bien utilisés également en mention puisque ce sont deux façons de dénommer le même référent. De plus, ils sont donnés comme lexique utilisé par deux émetteurs différents. 
(27) «Et puis, ajouta-t-elle dans un dernier mâchonnement enthousiaste, elle est si hartthhisstte !» (218)

'orthographe fantaisiste du mot suffit à signaler la particularité de la prononciation L'intégration de ce terme à un DD évite au narrateur de mettre des guillemets de distanciation. Il n'y a plus IT puisque le mot apparaît dans un discours totalement restitué même si la forme graphique essaie de rendre la forme sonore. Nous avons ici une sorte d'inverse de l'IT. Alors que l'IT laisse transparaitre les mots d'autrui dans ceux du narrateur, ici c'est le narrateur qui intervient dans la transcription de mots ne lui appartenant pas.

Enfin un autre morceau de bravoure concernant les particularités de prononciation des Cambremer, nous permet de montrer comment Proust s'amuse à noter la «noncoïncidence » entre les différents usages. Pour l'éditeur, ce paragraphe est un véritable casse-tête car il lui faut trouver des solutions différentes pour différencier IT, mention intégrant la prononciation, DD.

(28) Dans d'autres groupes mondains, quand on parlait des Chenouville(0), l'habitude était ( $\mathrm{du}$ moins chaque fois que la particule était précédée d'un nom finissant par une voyelle, car dans le cas contraire on était bien obligé de prendre appui sur le de (1), la langue se refusant à prononcer Madam'd'Ch'nonceaux(2)) que ce fut l'e (1)muet de la particule qu'on sacrifiât. On disait : « Monsieur d'Chenouville »(3). Chez les Cambremer la tradition était inverse, mais aussi impérieuse. C'était l'e muet de Chenouville(1bis) que dans tous les cas on supprimait. Que le nom fût précédé de mon cousin ou de ma cousine, c'était toujours de "Ch'nouville "(4) et jamais de Chenouville(4). (Pour le père de ces Chenouville on disait notre oncle(4) car on n'était pas assez gratin à Féterne pour prononcer notre «onk»(4), comme eussent fait les Guermantes dont le baragouin voulu, supprimant les consonnes et nationalisant les noms étrangers, était aussi difficile à comprendre que le vieux français ou le moderne patois.) Toute personne qui entrait dans la famille Cambremer recevait aussitôt, sur ce point des Ch'nouville(5), un avertissement dont Mlle Legrandin n'avait pas eu besoin. Un jour en visite, entendant une jeune fille dire : «ma tante d'Uzai »(3), « mon onk de Rouan »(3), elle n'avait pas reconnu immédiatement les noms illustres qu'elle avait l'habitude de prononcer : Uzès et Rohan(6); elle avait eu l'étonnement, l'embarras et la honte de quelqu'un qui a devant lui à table un instrument nouvellement inventé dont il ne sait pas l'usage et dont il n'ose pas commencer à manger. Mais la nuit suivante et le lendemain, elle avait répété avec ravissement : «ma tante d'Uzai »(3) avec cette suppression de l's (1)finale, suppression qui l'avait stupéfaite la veille, mais qu'il lui semblait maintenant si vulgaire de ne pas connaître qu'une de ses amies lui ayant parlé d'un buste de la duchesse d'Uzès (7), Mlle Legrandin lui avait répondu avec mauvaise humeur, et d'un ton hautain: «Vous pourriez au moins prononcer comme il faut: "Mame d'Uzai »(3). Dès lors elle avait compris qu'en vertu de la transmutation des matières consistantes en éléments de plus en plus subtils, la fortune considérable et si honorablement acquise qu'elle tenait de son père, l'éducation complète qu'elle avait reçue, son assiduité à la Sorbonne, tant aux cours de Caro qu'à ceux de Brunetière, et aux concerts Lamoureux, tout cela devait se volatiliser, trouver sa sublimation dernière dans le plaisir de dire un jour : « ma tante d'Uzai (3)».(213-214) 
(0) Il s'agit du désignateur employé sans "travail discursif» ou réflexion métalinguistique, contrairement à (3) ou (4).

(1) Les mots autonymes sont signalés par l'italique.

(1bis) Le mot est employé comme autonyme ; il devrait être en italiques.

(2) Nous avons ici un IT hypothétique qui essaie de rendre la prononciation mais aucun parti pris éditorial ne le signale.

(3) Les deux point et les guillemets après les verbes dire, répéter signale un DD comme en

(27) avec un essai de restitution de la prononciation, intervention du narrateur.

(4) Nous trouvons deux paires d'IT parallèles. Seul, l'IT fautif est entre guillemets. L'absence de guillemets du second nuit à la clarté du propos.

(5) L'IT n'apparaît plus comme tel. Le fragment discursif et sa prononciation sont totalement intégrés au discours du narrateur non qu'il fasse sienne cette prononciation mais pour symboliser le changement patronymique.

(6) Les deux patronymes ne sont que mentionnés ; la citation est démarquée par les deux points.

(7) L'usage du patronyme totalement intégré à la syntaxe du narrateur ne comporte pas, comme il se doit, de différence typographique.

Cet extrait illustre à deux titres l'emploi de l'IT. L'IT se présente comme un fragment discursif d'un locuteur secondaire intégré au discours du narrateur (4). Cette intégration se fait au niveau du discours premier, du discours indirect ou indirect libre. Il peut avoir un locuteur générique, "on»,ou spécifique: «chez les Cambremer». Comme toute modalisation, il comporte une part d'évaluation jusqu'à l'ironie, ici rendue par la présence ou non des guillemets. Chez Proust, l'intégration d'IT lui permet de restituer la parole d'un autre y compris dans ses particularités lexicales ou phoniques, de s'en amuser et d'en faire un élément supplémentaire de véracité dans la fiction.

Ironiquement, les cours du linguiste Brunetière sont rejetés par Mlle Legrandin au profit de l'accession à une caste marquée par l'acceptation de ses tics langagiers hors norme. La mention d'une prononciation spécifique marque la différence entre une simple Legrandin et une Cambremer ou mieux une Guermantes. L'initiation à la noblesse passe par la transformation de la mention en usage. La disparition de l'IT comme voix seconde, donc différente dans la langue de Mlle Legrandin est la métaphore du mouvement énonciatif qui diffère les voix (DD), les superpose (DIL), les inclut (IT), puis les intègre (DI) avant de les faire disparaître (DN).

\section{BIBLIOGRAPHIE}

AUTHIER-REVUZ, J. (1996) « Remarques sur la catégorie de l' « îlot textuel », Cahiers du français contemporain, « Hétérogénéités en discours, Crédif, Didier érudition : 91-115

AUTHIER-REVUZ, J. (1995) Ces mots qui ne vont pas de soi : Boucles réflexives et non-coïncidences du dire, Larousse, 2 vol., Paris.

DUCROT, O. (1984) Le dire et le dit, Minuit, Paris. 
ROSIER, L. (1999) Le discours rapporté, Histoire, théories, pratiques, Gembloux, Duculot.

TISSET, C. (2000) Analyse linguistique de la narration, chapitre $7:$ :La polyphonie énonciative », SEDES, Paris.

\section{NOTES}

1. Le terme de modalisation autonymique est employé par Authier-Revuz (1995) pour nommer toutes les formes de citations qui sont en usage et en mention. L'îlot textuel est une des formes de modalisations autonymiques.

\section{AUTEUR}

CAROLE TISSET

IUFM de Versailles

UMR 7114ParisX/CNRS Modyco 